STRUCTURAL BIOLOGY COMMUNICATIONS

ISSN 2053-230X

Keywords: second virial coefficient; crystallization; solubility; corrigendum.

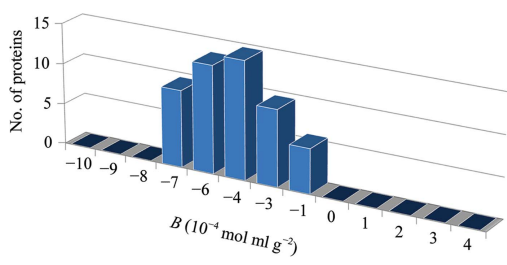

(C) 2016 International Union of Crystallography

\section{Applications of the second virial coefficient: protein crystallization and solubility. Corrigendum}

\author{
William W. Wilson ${ }^{a}$ and Lawrence J. DeLucas ${ }^{b}$
}

${ }^{\mathbf{a}}$ Mississippi State University, Starkville, MS 39759, USA, and ${ }^{\mathbf{b}}$ Center for Structural Biology, University of Alabama at Birmingham, 1720 Second Avenue South, Birmingham, AL 35294, USA. *Correspondence e-mail: duke2@uab.edu

A number of citations in the article by Wilson \& DeLucas [(2014). Acta Cryst. F70, 543-554] are corrected.

In the article by Wilson \& DeLucas (2014) there is an error in a citation which occurs several times. Tsumoto et al. (2005) is cited when the correct reference should be Hitscherich et al. (2000). This error occurs on page 548 in the first, third and last sentences of the third paragraph, and in the last sentence of the fifth paragraph. In each case Tsumoto et al. (2005) should be replaced by Hitscherich et al. (2000). The full reference for Hitscherich et al. (2000) is given below and the final three paragraphs on page 548 should read as follows:

Compelling examples of the use of $B$ values for membraneprotein crystallization have been reported in several publications (Kratochvil, 1987; Hitscherich et al., 2000; Berger et al., 2005, 2006; Bhat \& Timasheff, 1992). As noted earlier, before the advent of self-interaction chromatography for $B$ determinations it was extremely difficult to obtain such measurements via the traditional approach of static light scattering. The solution behavior of the bacterial outer membrane protein OmpF porin was studied by SLS in a variety of crystallization solutions (Hitscherich et al., 2000). B was demonstrated to be a clear predictor of the crystallization behavior of porin. Both tetragonal and trigonal porin crystals were found to form in a narrow range of $B$ values which were only located within the 'crystallization slot' as defined by Wilson for aqueous proteins. $B$ values were also used to study the effect of precipitants such as PEG on micelle size and interaction forces between micelles (Kratochvil, 1987; Hitscherich et al., 2000).

$B$ values for detergent micelles (free of protein) under identical crystallization conditions exhibited similar $B$ values to the protein-detergent complexes (PDCs). Thus, based on this one example using $\mathrm{OmpF}$, in which the detergent appears to dominate the interactions between PDCs, the authors suggest that for any given detergent membrane-protein crystallization screens may be designed by simply manipulating the detergent-solution properties until the measured $B$ values lie within the crystallization slot. This would minimize the amount of protein required for crystallization screening and improve productivity.

Studies performed by Berger and coworkers used measured second virial coefficient values to investigate 
protein interactions, leading to the crystallization of bacteriorhodopsin solubilized in $n$-octyl- $\beta$-D-glucoside (Berger et al., 2006). At low to moderate salt concentrations the PDC $B$ values show an increase in repulsive interactions followed by a sharp transition to attractive PDC interactions in a narrow range of high salt concentrations. PDC interactions 'were observed as the cloud-point temperature was approached for various salts', suggesting that the interaction trends are strongly influenced by the micelle structure and surfactant phase behavior, both of which are sensitive to salt and surfactant concentration. These data also suggest that for a PDC solution at a fixed surfactant concentration above its CMC the surfactant interactions may play a significant role in PDC interactions. Micelle shape and structure (influenced by the micelle concentration in specific salt concentrations) are also demonstrated to be important in PDC interactions. It was also demonstrated that for various combinations of additives and precipitants studied, attractive $\mathrm{PDC}$ interactions tend to occur at conditions approaching the $\mathrm{C}_{8} \beta \mathrm{G}_{1}$ cloud curve (the cloud point is the temperature or other variable change at which dissolved solids begin to precipitate, producing a cloudy appearance to the solution). This was observed previously for OmpF (Hitscherich et al., 2000).

\section{References}

Hitscherich, C. Jr, Kaplan, J., Allaman, M., Wiencek, J. \& Loll, P. J. (2000). Protein Sci. 9, 1559-1566.

Wilson, W. W. \& DeLucas, L. (2014). Acta Cryst. F70, 543-554.

Tsumoto, K., Ejima, D., Kita, Y. \& Arakawa, T. (2005). Protein Pept. Lett. 12, 613-619. 